Research Paper

International Journal of Medical Sciences

ISSN 1449-1907 www.medsci.org 2007 4(3):124-130

(C) Ivyspring International Publisher. All rights reserved

\title{
Relationships between free radical levels during carotid endarterectomy and markers of arteriosclerotic disease
}

\author{
Jagdish Gondalia 1, Björn Fagerberg 2, Johannes Hulthe 2, Lars Karlström ${ }^{3}$, Ulf Nilsson 4, Susanna Wa- \\ ters $^{5}$, and Olof Jonsson 1
}

1. Department of Urology, Sahlgrenska University Hospital, Göteborg, Sweden

2. Wallenberg Laboratory for Cardiovascular Research, Sahlgrenska University Hospital, Göteborg, Sweden

3. Department of Vascular Surgery, Sahlgrenska University Hospital, Göteborg, Sweden

4. The Renal Center, Department of Nephrology, Sahlgrenska University Hospital, Göteborg, Sweden

5. Department of Carlsson Research, P.O.B. 444, Göteborg, Sweden

Correspondence to: Prof. Olof Jonsson, Tel: +46 31 3421000; fax: +46 31 821740; E-mail: olof.jonsson@vgregion.se

Received: 2006.12.09; Accepted: 2007.04.13; Published: 2007.04.14

Background: Free radical production is elevated in jugular venous blood emerging from the brain in conjunction with carotid endarterectomy. This study explores the relationships between markers for lesion progression in arteriosclerosis, production of radicals and clinical characteristics.

Methods: The radical production during carotid endarterectomy was studied in 13 patients with an ex vivo spin trap method using OXANOH as a spin trap. MCP-1, ICAM-1, MMP-9 and oxLDL were determined in venous blood samples before, during and after clamping of the carotid artery. Principal component analysis (PCA) as well as partial least square regression analysis (PLS) was applied to interpret the data.

Results: PCA and PLS analysis revealed that high values of MMP-9 and low values of ICAM-1 were associated with high radical production whereas MCP-1 and oxLDL were not correlated to radical production. MMP-9 was elevated at diabetes, high haemoglobin, high leucocyte counts and thrombocyte counts as well as at contralateral stenosis, whereas ICAM-1 showed reversed relationships to these clinical variables. MCP-1 increased during surgery.

Conclusions: The main finding in our study is that MMP-9 in plasma is asscociated with radical production during carotid endarterectomy, suggesting that this enzyme might be involved in the pathogenesis of brain damage in conjunction with ischaemia-reperfusion.

Key words: Carotid endarterectomy; Free radicals; MCP-1; ICAM-1; MMP-9; oxLDL

\section{INTRODUCTION}

In previous studies we have described a technique to analyze the production of free radicals during carotid endarterectomy based on determination of radical production in vitro in venous blood samples $[1,2]$. The rationale behind this in vitro technique is that radical production continues in regional venous blood draining a previously ischaemic area due to e.g. activated leucocytes or ongoing lipid peroxidation. Pre-treatment of the patients with a xanthine oxidase inhibitor influences radical production, indicating that oxidation of accumulated hypoxanthine has a role to play in this process. The radical production is influenced by several clinical parameters, e.g. occurrence of contralateral stenosis, indicating that it is associated with the severity of the arteriosclerotic disease. There are several markers for the tissue damage characterising the lesion progression in arteriosclerosis, e.g. MMP-9, ICAM-1, MCP-1 and oxLDL.

Rupture of atherosclerotic plaques is a key process in symptomatic atherosclerosis. Fibrous caps from ruptured atherosclerotic plaques contain less ex- tracellular matrix than caps from intact plaques [3]. Enhanced matrix breakdown seems to be largely attributable to matrix-degrading metalloproteinases (MMP), which are expressed in atherosclerotic plaques, mainly by macrophages and foam cells [3]. The MMP family is catalytically active, both in vitro and in vivo. The activity of MMPs is increased by gene transcription, extracellular activation of the secreted inactive zymogen form, and reduced activity of tissue inhibitors of metalloproteinases. Latent MMPs can be activated by a number of molecules existing in the atherosclerotic plaque, of which several are produced by the macrophages, e.g. plasmin, reactive oxygen species and others [3]. Serum concentration of MMP-9 has been suggested as a biomarker of plaque vulnerability [4]. In diabetic patients, carotid artery plaques have more macrophages and higher levels of MMP-9 than non-diabetic patients [5].

The intercellular adhesion molecule ICAM-I mediates adhesion and transmigration of leucocytes to the vascular endothelial wall, a step suggested as being critical in the initiation and progression of atherosclerosis. Circulating ICAM-I is associated with a fu- 
ture risk of stroke [6] and is elevated in diabetic patients [7].

Monocyte chemoattractant protein-1 (MCP-I) originates from endothelial, epithelial, and glial cells and mediates recruitment of monocytes into tissues $[8,9]$.

Oxidised LDL (oxLDL) seems to be a key antigen in atherosclerosis and circulating levels of oxLDL have been observed to predict the development of carotid artery atherosclerosis [10,11].

Apart from atherosclerotic lesions, cerebral ischaemic damage may also cause increased radical production and expression of ICAM-1 and MCP-1, or be associated with increased MMP-9 production [12-16].

Accordingly, we explored whether there were any associations between the levels of MMP-9, MCP-1, ICAM-1, oxLDL, and free radicals in blood from the jugular vein, as well as variables related to atherosclerosis during endarterectomy.

\section{MATERIAL AND METHODS}

\subsection{Patients}

The 13 patients included in the present study were identical to the control group in our previous work concerning the effects of pre-treatment with a xanthine oxidase inhibitor on free radical levels during carotid endarterectomy in patients operated on for symptomatic carotid artery stenosis [2]. Relevant data concerning the patients are presented in Table 1.

TABLE 1. Characteristics of the patients

\begin{tabular}{lllllllllll}
\hline $\begin{array}{c}\text { Female/ } \\
\text { male }\end{array}$ & $\begin{array}{c}\text { Age (years) } \\
\text { median } \\
\text { range }\end{array}$ & Diabetes & Hypertension & $\begin{array}{c}\text { Cardiac } \\
\text { disease }\end{array}$ & Smoking & $\begin{array}{c}\text { Minor } \\
\text { stroke }\end{array}$ & $\begin{array}{c}\text { Amaurosis } \\
\text { fugax }\end{array}$ & $\begin{array}{c}\text { Left/ } \\
\text { TIA }\end{array}$ & $\begin{array}{c}\text { Degree of stenosis } \\
\text { right } \\
\text { (Mean } \pm \text { SEM) }\end{array}$ & $\begin{array}{c}\text { Contralateral stenosis } \\
\text { (Mean } \pm \text { SEM) }\end{array}$ \\
\hline $4 / 9$ & $72(58-81)$ & $4 / 13$ & $7 / 13$ & $5 / 13$ & $6 / 13$ & $8 / 13$ & $3 / 13$ & $2 / 13$ & $9 / 4$ & $89.6 \pm 1.4$ \\
\hline
\end{tabular}

\subsection{Methods}

\subsubsection{Anaesthesia}

The patients were premedicated with a combination of pethidine and dixyrazine (Esucos $\left.{ }^{\circledR}\right)$ according to their weight and were then operated on under fentanyl-assisted general anaesthesia.

\subsubsection{Operation}

The operation performed was described in detail in our previous article. Briefly, the carotid artery was exposed via an incision along the anterior border of the sternocleidomastoid muscle. A 1-mm catheter was inserted via the facial vein into the jugular vein and advanced up to the level above the skull base, and the facial vein was divided. The catheter was advanced $12-15 \mathrm{~cm}$ up until resistance and then withdrawn $1 \mathrm{~cm}$ until blood could be aspired without resistance. The common carotid and internal carotid artery were isolated separately without touching the carotid bifurcation, whereafter the external carotid artery was isolated.

After clamping the common and external carotid artery, stump pressure was measured using an arterial pressure monitor via a needle inserted into the internal carotid artery distal to the stenotic lesion. A shunt was not considered necessary in any case. After clamping the internal carotid artery, the internal and common carotid arteries were opened via a longitudinal arteriotomy and the plaque removed.

The arteriotomy was closed using 6-0 prolene continuous sutures. In two cases, in each group, the arteriotomy was closed using a patch to avoid narrowing of the internal carotid artery. There was no mortality and no cardiac or cerebral complications.

\subsubsection{Sampling procedure}

After declamping, blood flow was monitored using a sterile doppler probe and a flow meter (Medistim R, Vingmed, Sweden). From the catheter, inserted into the jugular vein, $4 \mathrm{~cm}^{3}$ blood samples were drawn three times at 5-minute intervals, before clamping the carotid artery, once one minute after clamping and once three minutes before declamping. Another four $4-\mathrm{cm}^{3}$ samples were drawn at 1, 5, 10 and 15 minutes after declamping. Due to the placement of the catheter it is unlikely that the blood samples were contaminated with blood from the external head.

After heparinisation, each venous blood sample was divided into two 1-ml portions (one sample and one blank) and pipetted into Eppendorf tubes. OXANOH (v.i.) (1 mM final concentration) was added to both tubes. In order to distinguish the part of the electronic spin resonance (ESR) signal attributable to superoxide and/or hydroxyl radicals, superoxide dismutase, catalase and desferrioxamine were added to the blank tube to a final concentration of $0.1 \mathrm{mg} \mathrm{ml}^{-1}$, 16,000 units $\mathrm{ml}^{-1}$ and $0.4 \mathrm{mg} \mathrm{ml}^{-1}$, respectively. The same volume of isotonic sodium chloride as used to solute the scavenger substances was added to the sample tube. Subtraction of the ESR signal seen in the samples treated with an antioxidant cocktail from that of the saline samples yields the part of the signal that can be attributed to superoxide and/or hydroxyl radicals or any secondary radicals dependent on these. The tubes were shaken and centrifuged at 14,000 rpm for one minute. The plasma was removed immediately and frozen in liquid nitrogen and the time from sampling to freezing was thus less than two minutes. 


\subsubsection{Measurement of radical production}

OXANOH was used as a spin trap. The stable nitroxide radical 2-ethyl-2,4,4-trimethyloxazolidine-3-3-yloxy (OXANO*) was reduced to the hydroxylamine spin trap 2-ethyl-3-hydroxy-2,4,4-trimethyloxazolidine (OXANOH) by gassing a $10 \mathrm{mM}$ solution of the radical with hydrogen for 45 minutes in the presence of the catalyst platinum dioxide $\left(\mathrm{PtO}_{2}\right)$. On contact with a radical, OXANOH is reoxidised to OXANO', which can be measured by ESR. The spin trap was freshly prepared before each experiment and kept on ice until used.

The samples were transported to the electronic spin resonance laboratory and thawed. The concentration of OXANO• was analysed using a Bruker ECS 106 ESR spectrometer. The spectrometer settings were as follows: field centre, $3478 \mathrm{G}$; modulation amplitude, 1 $\mathrm{G}$; microwave power, $10 \mathrm{~mW}$; microwave frequency, $9.70 \mathrm{GHz}$; scan range, $5 \mathrm{G}$; scan rate, $1 \mathrm{G} \mathrm{s}^{-1}$; time constant, $20 \mathrm{~ms}$. The ESR signal was quantified by signal height peak to trough.

The concentration of OXANO in the blank sample was subtracted from that of the corresponding test sample. Around ten per cent of the signal was diminished as a result of the inhibitors.

\subsubsection{Measurement of MCP-1, ICAM-1, MMP-9 and oxLDL}

Blood samples were withdrawn for an analysis of markers of arteriosclerosis together with OXANO samples 1, 5 and 9, i.e. before clamping, just before declamping and 15 minutes after start of reperfusion. The blood samples were heparinised and centrifuged, whereafter the plasma was frozen in liquid nitrogen for later analysis. MCP-1, ICAM-1 and MMP-9 were measured using commercially available ELISA kits from $R \& D$ systems, UK.

OxLDL concentration in plasma that had been stored at $-70^{\circ} \mathrm{C}$ was measured by a sandwich ELISA (Mercodia, Uppsala, Sweden) utilizing the same specific murine monoclonal antibody, mAb-4E6, as in the assay described by Holvoet et al [17]. Using this antibody (mAb-4E6) it is possible to measure very small amounts OxLDL containing a conformational epitope in the apoB-100 moiety of LDL that is generated as a consequence of substitution of lysine residues of apoB-100 with malondialdehyde. The specificity for the antibody mAb-4E6 has been assessed, showing that $50 \%$ inhibition of binding of mAb-4E6 to immobilized OxLDL was obtained with $0.025 \mathrm{mg} / \mathrm{dl} \mathrm{Cu}^{2+}$ OxLDL and with $25 \mathrm{mg} / \mathrm{dl}$ native LDL [18]. In the presently used sandwich ELISA, the plates are coated with the capture antibody (mAb-4E6), and the secondary antibody specifically detects apoB. In a set of 20 EDTA-plasma samples collected from 20 different individual blood-donors no unspecific binding of native LDL to the solid phase in the Mercodia oxidized LDL ELISA system was detected (data not shown). The precision has in our hands previously been shown to be satisfactory [19].

\subsection{Statistics}

The mean values for concentrations of OXANO and markers of arteriosclerosis before, during and after clamping are given. When analysing the relations between radical production and concentrations of MCP-1, ICAM-1, MMP-9 and oxLDL, mean values for all the OXANO concentrations measured during clamping and reperfusion and for the markers sampled before, during and after clamping were used. In the PLS models, where values for MMP-9 and ICAM-1 were compared to various parameters, mean values for the markers obtained before, during and after clamping were used.

Due to the small number of patients, standard descriptive statistics did not seem optimal. Instead, principal component analysis (PCA) was performed on the total data set. All variables were scaled to zero mean and unit variance. A detailed description of the computational steps involved in a PCA is given in Ref. 20. In essence, a variance/covariance matrix is calculated based on the scaled variables. Principal components are calculated as the eigen-vectors of this matrix, yielding the variable loadings shown in variable loading plots. The first principal component has the capacity to encompass a maximum of the variance in one single vector, which is a linear combination of all variables analysed. Each subsequent component constitutes an independent linear combination of variables, capturing a maximum of the variance remaining in the data set, and is orthogonal to all other components. In biological material, with a considerable degree of collinearity between the variables measured, the first component thus represents a large part of all information, compressed into one variable. The subsequent principal components represent independent information, in decreasing order of magnitude. In addition to reducing a large data set to a few components that can be easily overviewed, principal component-based analyses have important, inherent noise-reducing properties due to the simultaneous analysis of several variables. This is analogous to the reduction in noise gained by using large samples, where the large number of objects increases the precision of, for example, the sample mean. Results thus generated by PCA-based methods are more robust than corresponding univariate descriptors or bivariate correlation analyses. Compared with multiple regression techniques, the latter are highly sensitive to distribution and colinearities, while PCA can be applied to any kind of data, regardless of distribution, and, as outlined above, utilises the covariances to reduce noise and to compact the data. In subsequent analyses, Partial Least Square (PLS) regression analyses were applied [20]. As with PCA, the principal components are extracted with the modification that they are construed to find a regression between X- and Y-data in addition to producing a compact representation of the data. In the calculation of principal components, the components of the X-block that produce the strongest linear relation to the Y-variables are extracted. The principal difference is that in PLS, a number of (one or 
several) Y-variables are defined. For the regression coefficients yielded in PLS analyses, standard errors were estimated using the jack-knife procedure [21], which is a non-parametric, general principle for the estimation of errors in various estimates, suitable for PLS regression coefficients. All PCA and PLS models, as well as graphics and standard error estimates, were generated using the Simca-P 8.0 Software (Umetrics, Inc.).

Anova and paired T-test were used to test for differences in concentrations of MCP-1, ICAM-1, MMP-9 and oxLDL with time during the operations and $\mathrm{p}<0.05$ (two-sided) was considered statistically significant.

\section{RESULTS}

The mean values for all OXANO measurements performed during and after clamping were numerically higher than the baseline measurements although no statistically significant change was observed due to the great variations (Fig. 1).

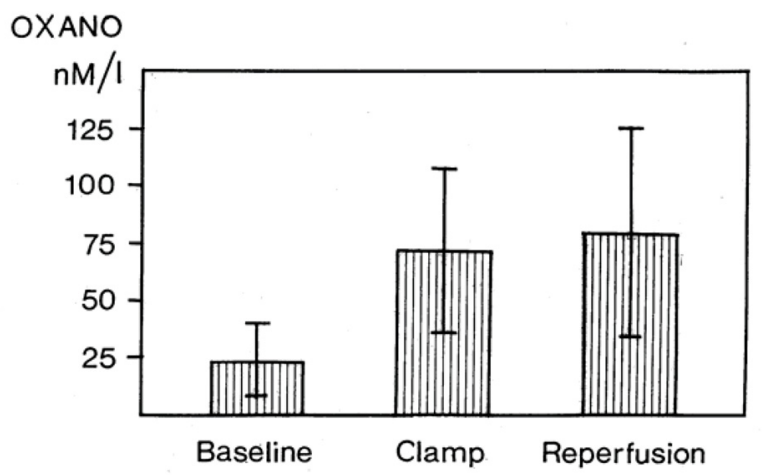

Fig. 1 Free radical production measured in vitro in blood samples from the internal jugular vein. Mean values \pm SEM are given for OXANO values obtained before clamp, during clamp and at reperfusion. $\mathrm{n}=13$.

The atherosclerosis markers show some changes during surgery (Fig. 2). MCP-1 increased significantly from the first time-point to the second and third ones, while oxLDL tended to decrease. No statistically significant change was observed concerning MMP-9 and ICAM-1.

The outcome of the PCA of the radical data, data concerning the degree of arteriosclerotic disease and some relevant clinical data are shown in Figure 3. In order to increase the lucidity of the figure the PCA has been modified by presenting the OXANO values as mean values before, during and after clamping the carotid artery. The OXANO radical levels (PL, I, RP) are located in a cluster at the far left of the X-axis together with the MMP-9 values, indicating a positive correlation between these variables. ICAM-1 values are located in the opposite direction, indicating a negative correlation with OXANO levels as well as MMP values. MCP-1 and oxLDL values are close to origin, indicating a lack of correlation between these values and any of the other variables.

MMP-9 values appear from this PCA to be positively correlated to e.g. platelets, leucocytes and dia- betes and negatively correlated to age, while the opposite situation appears to be true for ICAM-1 values.

A closer analysis of the significances of these relationships is given in the PLS analyses. The PLS regression analysis describing how OXANO levels depend on values for oxLDL, MMP-9, ICAM-1 and $\mathrm{MCP}-1$ is presented in Figure 3. As suggested from the PCA analysis, there is a strong positive correlation between MMP-9 and radical production and a negative correlation between ICAM-1 values and OXANO levels.

The regression coefficients relating various clinical parameters and MMP-9 or ICAM-1 values are shown in Figures 4 and 5 respectively.

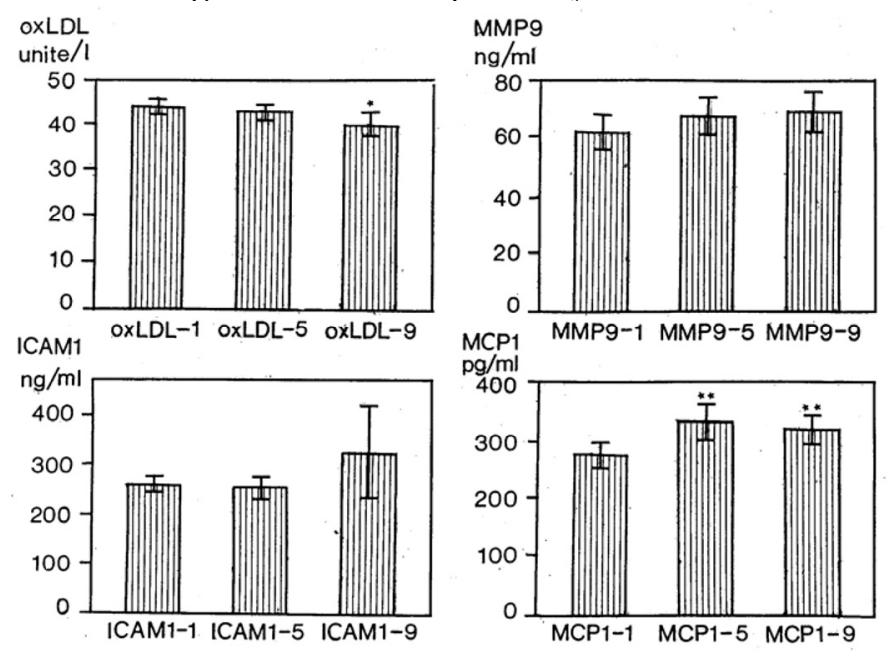

Fig. 2 Mean values (SEM) of oxLDL, MMP9, ICAM1 and MCP1 determined before clamping (1), during clamping (5) and 15 minutes after declamping (9). Stars denote significant differences compared to preclamp values. ${ }^{*} \mathrm{p}<0.05, * * \mathrm{p}<0.01$.

\section{PCA variable loading plot}

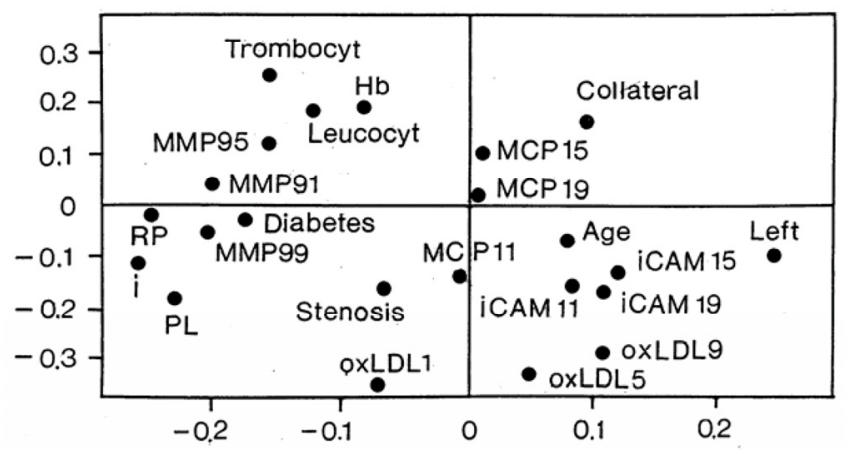

Fig. 3 Variable loadings derived from a PCA of some of the recorded data. The position of each variable in the loading plot indicates its relationship to other variables. Strongly correlated variables are located close to each other. Abbreviations: PL, I and RP OXANO levels at baseline, clamp and reperfusion respectively. MCP11, MCP15, MCP19; MCP levels before, during and after clamping, ICAM11, ICAM15, ICAM19; ICAM levels before, during and after clamping, MMP91, MMP95, MMP99; MMP9 levels before, during and after clamping, oxLDL1, oxLDL5, oxLDL9; oxLDL levels before, during and after clamping, Collateral; degree of collateral circulation, Stenosis; degree of ipsilateral stenosis, Left; operation for 
left-side stenosis, Diabetes; occurrence of diabetes, Leucocyt; white blood cell count, $\mathrm{Hb}$; haemoglobulin concentration, Trombocyt; trombocyte count.

Radical production vs arteriosclerosis markers

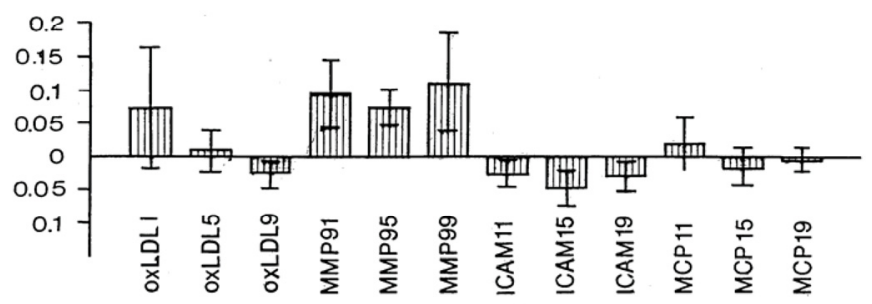

Fig. 4 Regression coefficients in a PLS model relating radical production to oxLDL, MMP9, ICAM1 and MCP1 determined before clamping (1), during clamping (5) and 15 minutes after declamping (9). Shown are scaled and centred regression coefficients with standard errors estimated by the jack-knife procedure. If the bars do not include the zero line a significant relationship prevails. A positive coefficient indicates a positive relationship to radical production and vice versa.

\section{MMP9 vs various parameters}

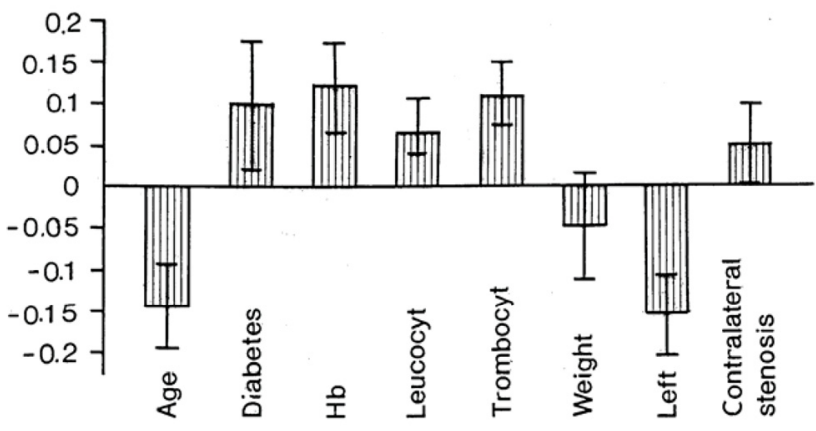

Fig. 5 Regression coefficients in a PLS model relating MMP9 levels to various clinical variables. Shown are scaled and centred regression coefficients with standard errors estimated by the jack-knife procedure. If the bars do not include the zero line a significant relationship prevails. A positive coefficient indicates a positive relationship to radical production and vice versa.

ICAM vs various parameters

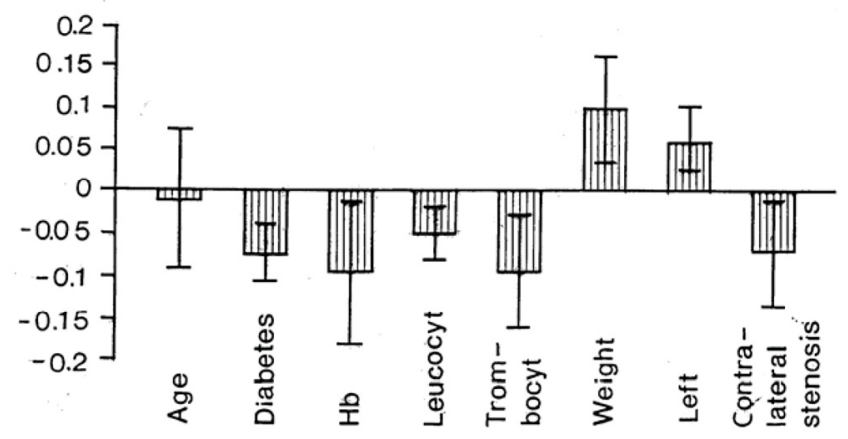

Fig. 6 Regression coefficients in a PLS model relating ICAM1 levels to various clinical variables. Shown are scaled and centred regression coefficients with standard errors estimated by the jack-knife procedure. If the bars do not include the zero line a significant relationship prevails. A positive coefficient indicates a positive relationship to radical production and vice versa.
Increased MMP-9 values are found in conjunction with diabetes, high haemoglobin, leucocyte and platelet values and at contralateral stenosis while low values are found in conjunction with high age, high blood pressure, operation for left-side stenosis and high stump pressure.

As regards ICAM-1, high values are found in conjunction with heavy weight, operation for left-side stenosis and high stump pressure. Low values are found in conjunction with diabetes, high haemoglobin, leucocyte and thrombocyte values and when a contralateral stenosis is present.

\section{DISCUSSION}

The present study concerns the relationships between the production of free radicals during and after operation for stenosis of the carotid artery and blood levels of ICAM-1, MMP-9, MCP-1 and oxLDL. Radical production has been calculated using an ex vivo spin trap technique with OXANOH as the spin trap. High values for MMP-9 and low values for ICAM-1 were associated with high levels of free radicals. Several correlations were found between the values of MMP-9 and ICAM-1 and different clinical variables. In an earlier study where the present group of patients were included as controls and compared to allopurinol-pretreated patients, relations between various clinical variables and radical production were reported [2].

MMPs are a group of zinc-dependent enzymes that degrade the molecules of the extracellular matrix. It has been shown that matrix degradation caused by these proteinases occurs during progression of atherosclerosis [22]. Furthermore, an increase in MMP activity occurs after stroke, which contributes to ischaemic brain injury, including infiltration of leucocytes in the damaged tissue [12-14]. In animal experiments it has been demonstrated that inhibition of MMP-9 reduces brain injury after stroke [23]. MMP-9 levels are increased in internal jugular venous blood after traumatic brain injury in patients [15]. These studies indicate that MMP-9 is involved in the pathogenesis of brain damage due to various causes. Our results of a positive correlation between levels of free radicals and MMP-9 at operation for carotid artery stenosis further support this role of metalloproteinases in brain tissue damage. The positive correlation between MMP-9 and leucocyte counts also appears logical since neutrophils can also produce these enzymes [14]. Our finding of a positive association between MMP-9 and diabetes fits in well with previous observations [5].

The occurrence of contralateral stenosis was associated with higher MMP-9 values, suggesting that a more severe atherosclerotic disease causes elevated MMP-9 levels. Inhibition of MMP-9 in conjunction with these operations might be useful to decrease possible brain damage.

The plasma concentration of ICAM-1 as well as other adhesion molecules is increased in conjunction with transient ischaemic attacks, indicating a central 
nervous system inflammatory reaction [16]. In the present study we found no correlation between MCP-1 and radical levels and a negative correlation between ICAM-1 and these levels. The lack of a positive correlation between radicals and these markers of inflammation, as well as between clinical parameters known to be associated with increased radical production, indicates that the possible hypoperfusion that occurs during carotid endarterectomy, and which is responsible for radical production, is not enhanced enough to cause cerebral inflammation, resulting in increased ICAM-1 and MCP-1 values. There was a significant increase in the concentration of MCP-1 during and after carotid endarterectomy compared with before clamping of the artery. Taken together, these findings indicate that the concentration of MCP-1 increases during surgery via a mechanism other than radical production.

One possible explanation for the lack of a positive correlation between ICAM-1 and radical production in the present series is that our patients suffered from late-stage atherosclerosis. In previous studies it has been shown that plasma levels of ICAM-1 are associated with sub-clinical femoral atherosclerosis in clinically healthy middle-aged men; and also with endothelial function in healthy young subjects $[24,25]$. Various stages of atherosclerosis in different patient populations might explain why various authors report different results regarding ICAM-1 and the risk of cerebrovascular complications. Elevated concentrations of ICAM-1 were reported to be associated with an increased risk of stroke [6] whereas no correlation was found between the expression of ICAM-1 and the severity of symptomatic carotid disease [26].

Oxidised, low-density lipoprotein (oxLDL) is found within the atherosclerotic blood vessels but not normal blood vessels [27]. OxLDL has an important role to play in atherogenesis since it attracts monocytes into the vascular intima and transforms them into foam cells [28]. Since blood is rich in a variety of antioxidants "fully oxidised LDL" does not exist in the circulation [28]. Nevertheless, small amounts of oxLDL are found in the circulation and a correlation between oxLDL and plaque occurrence in the carotid and femoral arteries has been demonstrated [11,19]. In the present study we did not find any association between radical production and oxLDL in plasma. However, it should be kept in mind that we only examined one of many epitopes that may be oxidized in LDL.

The limitation of the present study is that it included only patients with unstable carotid atherosclerotic plaques leading to symptomatic ischaemic disease, and that we had no reliable measure of the degree of damage to brain tissue. Hence, we can only interpret the associations between the markers studied and the free radical levels and factors related to atherosclerotic disease. Furthermore, it must be emphasised that the study is based on a limited number of patients, and the measurements are subject to a substantial variability. Thus, the study should be re- garded as hypothesis-generating rather than conclusive, with respect to the relationships observed between the variables collected.

We conclude that the major finding in the present study was that MMP-9 in plasma was related to the production of free radicals during carotid endarterectomy and also other variables known to co-variate with MMP-9, such as leucocyte count, diabetes and more extensive atherosclerosis. Furthermore, MCP-1 increased during surgery and this was not associated with radical formation.

\section{ACKNOWLEDGMENTS}

This study was supported by the Faculty of Medicine, Göteborg University, the Göteborg Medical Society and the Knut and Alice Wallenberg foundation. We want to thank Anita Fae for excellent laboratory and Elisabeth Ståhlgren for excellent secretarial work.

\section{CONFLICT OF INTEREST}

The authors have declared that no conflict of interest exists.

\section{REFERENCES}

1. Holm J, Nilsson U, Waters N, Waters S, Jonsson O. Production of free radicals measured by spin trapping during operations for stenosis of the carotid artery. Eur J Surg 2001;167:4-9.

2. Waters S, Fae A, Gondalia J, Holm J, Karlström L, Nilsson U, Jonsson O. Effects of pretreatment with a xanthine oxidase inhibitor on free radical levels during carotid endarterectomy. Free Rad Res 2004;38:283-93.

3. Shah P. Mechanisms of plaque vulnerability and rupture. J Am Coll Cardiol 2003; 41:15S-22S.

4. Loftus IM, Naylor AR, Bell PRF, Thompson MM. Plasma MMP-9 - a marker of carotid plaque instability. Eur J Vasc Endovasc Surg 2001;21:17-21.

5. Cipollone F, Iezzi A, Fazia M, Zucchelli M, Pini B, Cuccurullo C, et al. The receptor RAGE as a progression factor amplifying arachidonate-dependent inflammatory and proteolytic response in human atherosclerotic plaques: role of glycemic control. Circulation 2003;108:1070-7.

6. Tanne D, Haim M, Boyko V, Goldbourt U, Reshef T, Matetzky S, Adler Y, Mekori YA, Behar S. Soluble intercellular adhesion molecule-1 and risk of future ischemic stroke: a nested case-control study from the Bezafibrate Infarction Prevention (BIP) study cohort. Stroke 2002;33:2182-6.

7. Marfella R, Esposito K, Giunta R, Coppola G, De Angelis L, Farzati B, et al. Circulating adhesion molecules in humans: role of hyperglycemia and hyperinsulinemia. Circulation 2000;101:2247-51.

8. Takahashi K, Mizuarai S, Araki H, Mashiko S, Ishihara A, Kanatani A, Itadani H, Kotani H. Adiposity elevates plasma MCP-1 levels leading to the increased CD11b-positive monocytes in mice. J Biol Chem 2003;278:46654-60.

9. Yla-Herttuala S, Lipton BA, Rosenfeld ME, Sarkioja T, Yoshimura T, Leonard EJ, Witztum JL, Steinberg D. Expression of monocyte chemoattractant protein 1 in macrophage-rich areas of human and rabbit atherosclerotic lesions. Proc Natl Acad Sci USA 1991;88:5252-6.

10. Metso S, Loimaala A, Mercuri MF, Nenonen A, Vuori I, Oja P, Bond MG, Laine S, Rontu R, Lehtimaki T. Circulating oxidized low-density lipoprotein and common carotid artery intima-media thickness in a random sample of middle-aged men. J Biomed Sci 2004;11:356-61.

11. Wallenfeldt K, Wikstrand J, Fagerberg B, Hulthe J. Oxidized low 
density lipoprotein in plasma is a prognostic marker of subclinical atherosclerosis development in clinically healthy men. J Intern Med 2004;256:413-20.

12. Rosenberg GA, Navratil M, Barone F, Feuerstein G.Z. Proteolytic cascade enzymes increase in focal cerebral ischemia in rat. J Cereb Blood Flow Metab 1996;16:360-6.

13. Matsuo $Y$, Onodera $H$, Shiga $Y$, Nalcamur M, Ninomiya M, Kihara T, Kogure K. Correlation between myeloperoxidase-quantified neutrophil accumulation and ischemic brain injury in the rat: effects of neutrophil depletion. Stroke 1994;25:1469-75.

14. Weiss SJ, Peppin GJ. Collagenolytic metalloenzymes of the human neutrophil. Biochem Pharmacol 1986;35:3189-97.

15. Suehiro E, Fujisawa H, Akimura T, Ishihara H, Kajiwara K, Kato S, Fujii M, Yamashita S, Maekawa T, Suzuki M. Increased matrix metalloproteinase-9 in blood in association with activation of interleukin-6 after traumatic brain injury: influence of hypothermic therapy. J Neurotrauma 2004;21:1706-11.

16. Selakovic V, Colic M, Jovanovic M, Raicevic R, Jovicic A. Cerebrospinal fluid and plasma concentration of soluble intercellular adhesion molecule 1, vascular cell adhesion molecule 1 and endothelial leukocyte adhesion molecule in patients with acute ischemic brain disease. Vojnosanit Pregl 2003;60:139-46.

17. Holvoet P, Vanhaecke J, Janssens S, Van de Werf F, Collen D. Oxidized LDL and malondialdehyde-modified LDL in patiens with acute coronary syndromes and stable coronary artery disease. Circulation 1998;98:1487-94.

18. Holvoet P, Donck J, Landeloos M, Brouwers E, Luijtens K, Arnout J, Lesaffre E, Vanrenterghem Y, Collen D. Correlation between oxidized low density lipoproteins and von Willebrand factor in chronic renal failure. Thromb Haemost 1996;76:663-9.

19. Hulthe J, Fagerberg B. Circulating oxidized LDL is associated with subclinical atherosclerosis development and inflammatory cytokines (AIR Study). Arterioscler Thromb Vasc Biol 2002;22:1162-7.

20. Martens H, Naess T. Multivariate calibration. New York: Wiley. 1989.

21. Miller RG. The jackknife - a review. Biometrika 1974;61:1-17.

22. Galis ZS, Sukhova GK, Lark MW, Libby P. Increased expression of matrix metalloproteinases and matrix degrading activity in vulnerable regions of human atherosclerotic plaques. J Clin Invest 1994;94:2493-503.

23. Romanic AM, White RF, Arleth AJ, Ohlstein EH, Barone FC. Matrix metalloproteinase expression increases after cerebral focal ischemia in rats. Stroke 1998;29:1020-30.

24. Holmlund A, Hulthe J, Millgard J, Sarabi M, Kahan T, Lind L. Soluble intercellular adhesion molecule- 1 is related to endothelial vasodilatory function in healthy individuals. Atherosclerosis 2002;165:271-6.

25. Hulthe J, Wikstrand J, Mattsson-Hultén L, Fagerberg B. Circulating ICAM-1 (intercellular cell-adhesion molecule 1) is associated with early stages of atherosclerosis development and with inflammatory cytokines in healthy 58-year-old men: the Atherosclerosis and Insulin Resistant (AIR) study. Clin Sci (Lond) 2002;103:123-9.

26. Nuotio K, Lindsberg PJ, Carpen O, Soinne L, Lehtonen-Smeds EM, Saimanen E, Lassila R, Sairanen T, Sarna S, Salonen O, Kovanen PT, Kaste M. Adhesion molecule expression in symptomatic and asymptomatic carotid stenosis. Neurology 2003;60:1884-5.

27. Tsimikas S, Witztum JL. Measuring circulating oxidized low-density lipoprotein to evaluate coronary risk. Circulation 2001;103:1930-2.

28. Silaste ML, Rantala M, Alfthan G, Aro A, Witztum JL, Kesäniemi YA, Hörkkö S. Changes in dietary fat intake alter plasma levels of oxidized low-density lipoprotein and lipoprotein(a). Arterioscler Thromb Vasc Biol 2004;24:498-503. 